\title{
REGGENZA E ACCORDO
}

1. I rapporti esistenti fra le parole nella frase possono raggrupparsi, com'è noto, in tre categorie: reggenza, funzione e ordine delle parole.

La reggenza riguarda il modo in cui alcune parole impongono ad altre certe varizioni di forma (genere, numero, caso, persona). Rientra in questa categoria anche I' accordo.

La funzione può definirsi quel rapporto particolare che unisce un elemento costitutivo (un tassema) alla totalità della frase (ovvero all' elemento reggente di un sintagma).

L'ordine delle parole comprende le regole che soprassiedono alla sequenza dei "segmenti locutori" nel discorso.

Questi tre aspetti della sintassi possono venir trattati indipendentemente l'uno dall'altro, tuttavia il concetto di reggenza è talmente legato a quello di funzione che relativi richiami fra questi due concetti riescono inevitabili.

2. Partiremo dalla distinzione dei due concetti di sintagma e di tassema. ${ }^{1}$

Il sintagma è la connessione o combinazione di due segni correlativi di cui uno è l'elemento reggente e l'altro un elemento dipendente. Esso non ha funzioni nella frase.

Il tassema è un elemento costitutivo della frase, portatore di una funzione logico-sintattica.

Formalmente i due concetti possono anche coincidere. Così nella frase:

\section{Abbiamo appreso una bella notizia}

il gruppo "una bella notizia" preso a sé è un sintagma che qui, nella frase, acquista il valore di un tassema, e precisamente di un oggetto diretto complesso, dipendente dal predicato verbale "abbiamo appreso". L'oggetto diretto "una bella notizia" è complesso perché contiene un attributo (bella). Abbiamo quindi il caso, ricordato più sopra, di un tassema (bella) dipendente dall'elemento reggente di un sintagma ("una notizia").

Anticipando ora alcuni dati che saranno sviluppati in seguito diremo che nella frase citata più sopra riscontriamo una reggenza verbale, quella del verbo apprendere che regge un complemento diretto (ossia un oggetto diretto); e abbiamo 
pure un caso di accordo (o concordanza): l'attributo "bella" che concorda col suo elemento reggente ("notizia") in genere, numero (e "caso").

3. Essendo i concetti di reggenza e accordo strettamente legati alla nozione di tassema, presentiamo a titolo orientativo un elenco dei tassemi stessi rimandando il lettore per notizie più ampie all'opera citata qui in nota. ${ }^{2}$ I tassemi sono in tutto dieci:

1. Soggetto

2. Predicato $\left\{\begin{array}{l}\text { verbale }(2.1) \\ \text { nominale }(2.2) \\ \text { predicativo } \\ \text { legato }(2.3)\end{array}\right.$

3. Oggetto diretto

4. Oggettoide (con una ventina di sottospecie)

5. Avverbiale. (Domanda sintattica: come?)
6. Circostanziale: dove?, quando?, perché?, a che scopo?

7. Giudicativo

8. Predicativo libero

9. Attributo

10. Apposizione.

Ogni tassema è contrassegnato da un numero-base che lo simboleggia. Questo numero può venir ulteriormente specificato con altre indicazioni e assumere così la funzione di un dettagliato indicatore simbolico. Tutto cio con l'intento di rendere più funzionali e rapide le analisi sintattiche, i raffronti contrastivi, le versioni in altre lingue. 3

4. La reggenza può esser definita anche come "la capacità che una parola possiede di governare, ossia reggere, un'altra parola". Invertendo i termini, la reggenza puo definirsi inoltre come "dipendenza sintattica di una parola da un" altra".

Una tale connessione o combinazione di parole in cui distinguiamo un elemento reggente e uno dipendente costituisce, come sappiamo, un sintagma. Tuttavia il concetto di sintagma è più ampio perché comprende anche la nozione di accordo.

A seconda dell' appartenenza morfologica dell'elemento reggente, le reggenze si dividono in verbali, sostantivali, aggettivali, pronominali e avverbiali.

L'elemento dipendente ha la funzione di un tassema e púo essere giustapposto, come nel caso dell'oggetto diretto. Parliamo allora di reggenza diretta. Se invece l'elemento dipendente è introdotto da preposizione, esso costituisce una reggenza indiretta. In linea di massima appartengono alle reggenze indirette l'oggettoide, l'avverbiale e il circostanziale.

Gli attributi sono parte reggenze indirette (attributi sostantivali!), parte reggenze dirette (attributi giustapposti!). Rientrano invece nella categoria dell' accordo gli attributi aggettivali (come si vedrà piu avanti). 
Il giudicativo e l'apposizione, tassemi ritmicamente indipendenti, sono in genere giustapposti.

Il soggetto, che ha funzione di elemento reggente, non viene introdotto da preposizione fuorché nel caso del partitivo. 4

Il predicato e $\mathrm{i}$ due predicativi rientrano nella sfera dell'accordo (v. il paragr. n. 7).

5. Per un altro verso, non puramente morfologico, le reggenze si classificano in forti e deboli.

Vengono considerate forti le reggenze, sia dirette che indirette, condizionate da fattori grammatico-lessicali e semantici dell'elemento reggente; e sono: 1'oggetto diretto, l'oggettoide (o oggetto preposizionale) e certi attributi non-aggettivali. Nelle reggenze forti la preposizione (in quanto è presente) è strettamente legata all'elemento reggente. Questo è uno dei criteri distintivi delle reggenze forti. (V. gli esempi al n. 6).

Si considerano deboli le reggenze tassematiche costituenti casi di libero raccordo o connessione.

Allo stato attuale delle ricerche non è sempre facile scindere in modo netto i casi di reggenza forte da quelli di reggenza debole. Se la cosa appare chiara nel caso degli oggetti diretti (tutti reggenze forti), la classificazione diventa alle volte meno agevole quando si tratta di distinguere fra loro oggettoidi (reggenze forti) e circostanziali (reggenze deboli) e talora anche avverbiali (pur essi reggenze deboli). Diverso è infatti il valore sintattico di due costrutti come:

a) Confidiamo nelle proprie capacità (oggettoide di contenuto)

e

Rimarremo nelle proprie abitazioni (cirostanziale di luogo)

b) Abbiamo provveduto a tutto (oggettoide dativale)

e

Procureremo di tornare all' alba (circostanziale di tempo)

c) Abbiamo dormito con le finestre aperte (circostanziale di concomitanza)

Ci hanno trattato con modi aspri (avverbiale di modo)

In casi isolati ci troviamo talora di fronte a vere e proprie bivalenze tassematiche. ${ }^{5}$ Due esempi potranno illustrare il fenomeno:

Largheggia in promesse - (promette molto : oggettoide)

(è generoso, come? : avverbiale) 


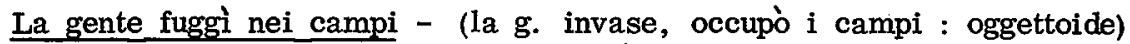

(la g. fuggì dove? : circostanz.)

Per arrivare a soluzioni più sicure in casi del genere, ci vorranno ricerche esaurienti sulle valenze verbali, e non solo : occorreranno in genere studi approfonditi sulla sintassi dei semi che possano rivelare tutta la ricchezza semantica di una parola e quindi anche le sue possibili reggenze. ${ }^{6}$

6. Cerchiamo ora di illustrare i vari tipi di reggenza con esempi concreti. Inizieremo con le reggenze forti:

\section{Oggetti diretti}

Ricordo quella serata.

Abbiamo discusso il problema.

Ho letto ambedue gli articoli.

Compreremo dei fiori.

Hanno schivato il pericolo.

\section{Oggettoidi}

$\mathrm{Ci}$ atterremo alle istruzioni. Hanno discusso di macchine. $\mathrm{Si}$ specializza in fisica. Abbiamo camminato per i campi. Ho girato per la piazza. Parliamo d'altro.

\section{Doppie reggenze}

(oggetto + oggettoide o viceversa)

Lo abbiamo ringraziato del favore.

Gli hanno offerto un posto.

Ci hanno privato di tutto.

Si è disgustato della pittura primitiva.

Lo hanno destituito da direttore.

Oggetti e oggettoidi sono tassemi verbali perché dipendenti da verbi. Alla categoria delle reggenze forti appartengono anche alcuni tassemi nominali, e precisamente quelli che sono condizionati dall' elemento reggente. Trattasi praticamente di quegli attributi sostantivali in cui la preposizione è strettamente legata al primo elemento del sintagma, rappresentato in questi casi da un sostantivo o da un aggettivo.

\section{Attributi oggettivi}

la spedizione del pacco

la consegna del diploma

il conferimento del titolo

la promessa di un premio

la costruzione di motori

i ricordi dell' infanzia

il desiderio d'affetto

la sete di potere
Attributi dipendenti da aggettivi transitivi

favorevole agli accordi desideroso di affermarsi memore dei benefici gradito al palato capace di resistere difficile a credere propenso a cedere contento della soluzione 
Appartengono invece alle reggenze deboli i numerosi altri attributi sostantivali (di luogo, tempo, materia, argomento, ecc.). Essi non sono determinati da caratteri grammaticali, lessicali o semantici dell'elemento reggente. La preposizione è legata in questi casi all'elemento dipendente.

Ci limitiamo a pochi esempi rinviando anche qui alla grammatica citata (v. nota 2):
i vini di Dalmazia una casa sulla collina una foresta nel crepuscolo una bottiglia di liquore

\author{
un oratore dalla parola facile \\ una lezione di elettronica \\ un anello d'oro \\ un concerto di musica romantica
}

Altre reggenze deboli sono rappresentate dai due tassemi verbali, l'avverbiale e il circostanziale. Anche qui la preposizione, quando c'è, è legata all'elemento dipendente.
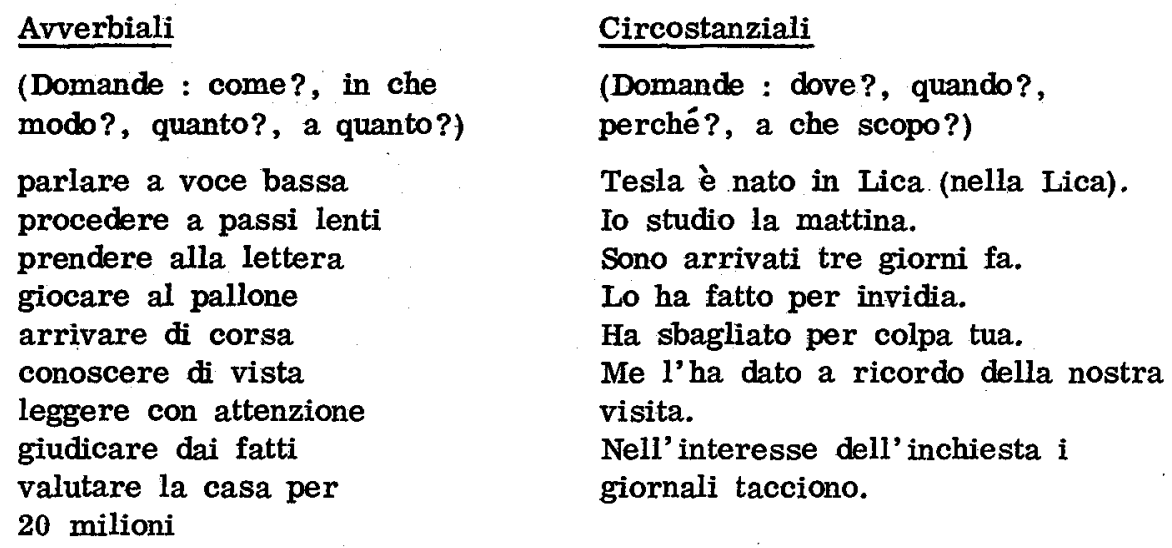

7. Dalla reggenza, come nesso sintattico, va distinto l'accordo (o concordanza). Mentre nel caso della reggenza l'elemento dipendente resta invariato tutte le volte che il termine reggente subisce variazioni morfologiche, l'accordo comporta il mutamento parallelo di ambedue $i$ termini del nesso.

Rientrano nella categoria dell' accordo innanzi tutto gli aggettivi e i participi nella funzione di attributi. Essi concordano con il termine reggente in genere, numero (e caso):
un lavoro meritevole
un campo lavorato
due lavori meritevoli
alcuni campi lavorati

una notizia importante

delle notizie importanti 
La concordanza riguarda poi il verbo coniugato (e il suo elemento nominale) che si accorda col soggetto in persona e numero.

Possiamo distinguere tre casi:

a) Soggetto e predicato verbale:

$$
\text { Egli parte stasera. - Essi partono stasera. }
$$

b) Soggetto e predicato nominale:

$$
\text { Egli è contento. - Essi sono contenti. }
$$

c) Rientrano nella sfera dell'accordo anche i due predicativi quando sono espressi da aggettivi o sostantivi. Essi si accordano col loro soggetto o oggetto in persona e numero:
Egli sembra sorpreso.
Il ragazzo si è fatto adulto.
Essi sembrano sorpresi.
I ragazzi si son fatti adulti.
Ella si confessó colpevole.
Lo promossero colonnello.
Esse si confessarono colpevoli.
Li promossero colonnelli.
Il tempo scorreva rapido.
Lo vide passar veloce.
Le ore scorrevano rapide.
Li videro passare veloci.

Il presente contributo vorrebbe costituire lo schema per una classificazione razionale delle due categorie sintattiche finora poco trattate, la reggenza e l'accordo. Formulo 1'augurio che " 1 'articolo possa servire da incentivo per ulteriori approfondimenti del problema.

\section{Note}

1 Quanto alla distinzione di questi due concetti e al loro vario sviluppo semantico mi permetto di rinviare al mio articolo "Tassemi e sintagmi" in SRAZ 23 (1969), pp. 81-85. Il termine tassema nel significato di elemento funzionale della frase è stato accolto nel frattempo dal compianto Giacomo Devoto nel suo ultimo lavoro Lezioni di sintassi prestrutturale, Firenze 1974.

2

I tassemi come elementi funzionali della frase sono trattati ampiamente nella Grammatica italiana descrittiva di M. Regula e J. Jernej, Francke, Berna $1975 .^{2}$

3

V. a tal proposito il mio articolo " $L$ ' analisi tassematica e le sue applicazioni" in SRAZ 39 (1976), pp. 27-37.

4 Com'è noto, anche l'oggetto diretto può essere rappresentato da un partitivo.

5 Cfr. il mio articolo "Intorno alle bivalenze tassematiche". Sta in Scritti in onore di Giuliano Bonfante. Paideia, Brescia (1975), pp. 383-388.

6 Sorin Stati, "Analisi componenziale: la sintassi dei semi". Sta in Teoria e storia degli studi linguistici. Roma 1975 (SLI), pp. 409-415. 
REKCIJA I KONGRUENCIJA

Članak obradjuje odnose sintaktičke rekcije prema kongruenciji. Problem se povezuje $s$ analizom funkcionalnih dijelova rečenice, a sve je praćeno s primjerima iz talijanskog jezika. 